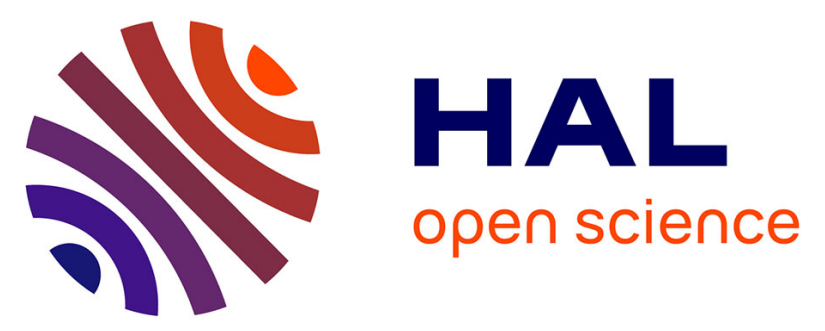

\title{
Multi-objective Optimisation of Wireless Sensor Networks Deployment: Application to fire surveillance in smart car parks
}

Slimane Charafeddine Benghelima, Mohamed Ould-Khaoua, Ali Benzerbadj,

Oumaya Baala

\section{To cite this version:}

Slimane Charafeddine Benghelima, Mohamed Ould-Khaoua, Ali Benzerbadj, Oumaya Baala. Multiobjective Optimisation of Wireless Sensor Networks Deployment: Application to fire surveillance in smart car parks. International Wireless Communications and Mobile Computing Conference, Jun 2021, Harbin, China. hal-03549340

\author{
HAL Id: hal-03549340 \\ https://hal.science/hal-03549340
}

Submitted on 31 Jan 2022

HAL is a multi-disciplinary open access archive for the deposit and dissemination of scientific research documents, whether they are published or not. The documents may come from teaching and research institutions in France or abroad, or from public or private research centers.
L'archive ouverte pluridisciplinaire $\mathbf{H A L}$, est destinée au dépôt et à la diffusion de documents scientifiques de niveau recherche, publiés ou non, émanant des établissements d'enseignement et de recherche français ou étrangers, des laboratoires publics ou privés. 


\section{Multi-objective Optimisation of Wireless Sensor Networks Deployment: Application to fire surveillance in smart car parks}

\author{
Slimane Charafeddine Benghelima, Student Member, IEEE \\ LRDSI \\ Department of Computer Science, University of Blida 1 \\ Blida, Algeria \\ benghelima.slimane@etu.univ-blida.dz
}

\author{
Mohamed Ould-Khaoua \\ LRDSI \\ Department of Computer Science, University of Blida 1 \\ Blida, Algeria \\ mouldkhaoua@univ-blida.dz
}

\author{
Oumaya Baala \\ FEMTO-ST Institute, Univ. Bourgogne Franche-Comté, CNRS \\ UTBM, Belfort, France \\ oumaya.baala@utbm.fr
}

\author{
Ali Benzerbadj \\ Department of Mathematics and Computer Science \\ Ain Temouchent University Belhadj Bouchaib \\ Ain Temouchent, Algeria \\ Research Laboratory in Industrial Computing \\ and Networks (RIIR), Oran, Algeria \\ ali.benzerbadj@univ-temouchent.edu.dz
}

\begin{abstract}
The exponential growth of the Internet-of-Things (IoT) technology paradigm has resulted in new applications and on-line services. Smart car park is one interesting example among others that can take advantage of applications based on wireless sensor networks (WSNs) Which constitute the core of IoT. This paper focuses on the deployment optimization problem of WSNs dedicated to the fire detection in a smart car park. In such networks, the nodes are classified into two categories: Sensor Nodes (SNs) deployed within the smart car park for targets coverage and Relay Nodes (RNs) whose task is to relay alert messages generated by the sensor nodes up to the sink node. In this study, we propose a Multi-Objective Binary Integer Linear Programming (MOBILP) which minimizes simultaneously the number of sensor nodes, relay nodes and the maximum distance from sensor nodes to the sink node, while ensuring coverage and connectivity. We have conducted extensive tests in order to evaluate the performance of our proposal. The results demonstrate that the MOBILP outperforms the existing approaches in terms of quality of solutions compared to a sequential deployment method, which consists to deploy SNs then RNs, and in terms of the ability to find other efficient solutions compared to a simultaneous deployment method using a mono-objective function, which consists to deploy SNs and RNs simultaneously.
\end{abstract}

Index Terms-Internet of Things, Wireless Sensor Networks, Deterministic Deployment, Multi-objective Combinatorial Optimization, Smart Car Parks, Fire Surveillance.

\section{INTRODUCTION}

The deployment of IoT technologies has recently intensified. The IoT paradigm refers to a set of technologies designed to interconnect physical devices with each other and with the Internet [2]. A key feature of IoT systems is that the network is composed of nodes with constrained resources, i.e., processing, communication, and storage. In this work, we consider WSNs, which are the core of such systems, composed of SNs, RNs, and sink nodes. A SN's primary role is to collect information from the surrounding environment and send them to the sink node directly or through the RNs. As a typical part of a smart city application, a smart car park [11] is a typical example of how the IoT technology can be broadly applied to deliver various services to different users in our everyday living environments.

Smart park helps drivers quickly find a free space in the nearby parking lot. In various parking lots, to determine the availability or unavailability of a car park spot, sensors are placed on the ground [14], demanding installing one sensor at each car parking spot, which could be costly, particularly in large parking lots. Motivated by this observation, various approaches have focused on using a camera-based WSN [9] to determine the state of the car park spots and can also be used for other services like surveillance. Such a parking lot is a sensitive area that presents a considerable risk to cars due to, e.g., fire incidents, as seen in table I; some recent car fires, which has caused damage to a massive number of cars, are cited. This has motivated us to design an effective targets (car park spots) surveillance system to avoid such losses, that can be a source of human tragedy and loss of the insurance company's earnings.

In this paper, we are interested in the problem of the deterministic deployment of WSNs [6]. A number of techniques have been developed to tackle this problem [1]. Generally, the existing studies have focused on placing the SNs [4] and then the RNs [16] separately with the aim of reducing deployment cost while maintaining low network delay and energy consumption. In this study, we strongly argue that the 
TABLE I

CAR PARK FIRE STATISTICS

\begin{tabular}{|c|c|c|}
\hline Fire Date & Car park Fire places & $\begin{array}{c}\text { Number } \\
\text { of damaged cars }\end{array}$ \\
\hline 2020 & Norway airoport & +1000 \\
\hline 2020 & Southwest Florida International Airport & +3500 \\
\hline 2019 & Aero India Show & +300 \\
\hline 2017 & Liverpool car park & +1000 \\
\hline 2016 & Winchester, England & 80 \\
\hline
\end{tabular}

simultaneous placement of both SNs and RNs may constitute a better alternative over the existing solutions for the deployment of WSNs. Indeed, we demonstrate through a series of tests that the simultaneous placement of both SNs and RNs offers a better alternative over existing solutions reported in the literature.

This study focuses on the simultaneous placement of SNs and RNs using a multi-objective formulation, where the objectives are the minimization of: the number of SNs, the number of RNs and the diameter ${ }^{1}$ of the network, under the coverage and connectivity constraints. It is noteworthy that in our case, the minimization of the diameter is considered in order to enhance the end to end delay of the alert delivery.

The rest of this paper is organized as follows. Section II presents the related works on WSN deployment problem. Sections III and IV provide respectively the network description and assumptions, and the problem formulation. Section $\mathrm{V}$ presents the MOBILP formulation. Section VI details the experimental findings. Section VII concludes the paper.

\section{RELATED WORKS}

In the following, we review some works related to the deterministic deployment problem of WSNs.

The authors in [5] addressed the deployment strategies that consider multi objectives such as cost (number of sensors), coverage, network connectivity and lifetime. To do so, they formulated two variants of a mixed-integer linear program (MILP) model. As a first step, they solve the problem of monitoring targets using a minimum number of connected sensor nodes. Then, the above formulation is extended to another MILP called lifetime-aware model, which considers the requirement that the WSN must remain fully operational for a prescribed time period $\mathrm{T}$.

The authors in [7] proposed a Linear program (LP) that considers $\mathrm{k}$ targets and a set of candidate positions for sensor nodes. It aims to place an optimal number of sensor nodes while satisfying k-Coverage (each target is covered by at least $\mathrm{k}$ sensor nodes) and $\mathrm{m}$-connectivity (each sensor node has at least m-neighbor nodes). The authors proposed a metaheuristic algorithm using the Bio-geography-based optimization to solve the problem. They built a multi-objective function from the weighted sum of the three objective functions: maximize target coverage, maximize the connectivity of each sensor node and minimize the total number of sensor nodes.

\footnotetext{
${ }^{1}$ Represents the length of the longest path among all shortest paths between all the SNs and the sink node
}

In both the previous cases, the SNs are responsible for sensing real world events and sending them to the sink node, which consume a lot of energy. Furthermore, since SNs have limited battery power and transmission range, the multi-hop communication is required in most situation. Therefore, SNs participate in the data routing to the sink node, which causes the rapid exhaustion of the SNs' batteries and increases the overall packet delivery latency.

To face the problems mentioned above, a new solution is introduced which consists of using RNs instead of SNs to relay data from SNs up to the sink node. The authors in [8] focused on the minimization of the number of SNs and RNs. They used a sequential approach, where the first stage ensures the Target Coverage (TC), and the second stage ensures Network Connectivity (NC) and Fault Tolerance (FT). The TC problem is proved to be an NP-hard problem and consequently a nonexact greedy algorithm is utilized to achieve full coverage. The second stage, known as Minimum RN Placement was reduced to the Minimum Euclidean Steiner Tree problem (MEST). MEST is known to be NP-hard, and thus a new heuristic algorithm based on the traveling salesman Problem is used to find two distinct paths from any SN to the sink node. In [12], in order to cope with the problem of TC and NC, a sequential approach is adopted. First, a greedy coverage algorithm places the sensors to cover all targets by assigning one sensor to each target. Second, a redundancy suppression algorithm removes redundancies resulting from the placement in the first step in order to reduce the number of deployed sensors. The last step is to place RNs to generate a connected graph with a minimum path length that connects the deployed sensors to the receiving node.

It is noteworthy that both works [8][12] rely on the sequential node placement approach where the SNs are deployed first, then the RNs based on the prefixed SNs. This approach does not explore the research space well to find better solutions. Therefore, the authors in [15] formulated the minimum-cost node placement problem as a Steiner Tree Problem (STP) for the simultaneous placement of SNs and RNs. They highlighted the benefits of this approach over the sequential node placement. The STP is further extended to Node Weighted Full Group Steiner Tree Problem and solved using an exact algorithm (for small instances) and a heuristic algorithm (for large instances). Afterwards, the sub-optimal solution obtained from the heuristic algorithm is improved through post-processing algorithms. To the best of our knowledge, the paper [15] is the only existing study on the simultaneous placement of SNs and RNs nodes using a mono-objective function which gets only one solution, whereas the number of $\mathrm{SNs}$ and $\mathrm{RNs}$ are two conflicting objectives. Hence, the multi-objective optimization is more suitable for such a problem to find all compromises.

In the current work, we are addressing the multi-objective optimization of the simultaneous placement of SNs and RNs.

\section{NETWORK DESCRIPTION AND ASSUMPTIONS}

As depicted on Figure 1, a WSN is composed of two fundamental sets, namely a set of SN (sensor node with dome 
camera) and a set of RN. The SNs have the role of capturing images of the spots inside the parking lot, and processing them locally using intelligent algorithms [10] to obtain information on the state of car park spots (free or occupied parking places), and whether there is a fire in the cars parked in the spots. The RNs are used to relay data packets (spots status or fire alarm) generated by the SNs, up to the sink node.

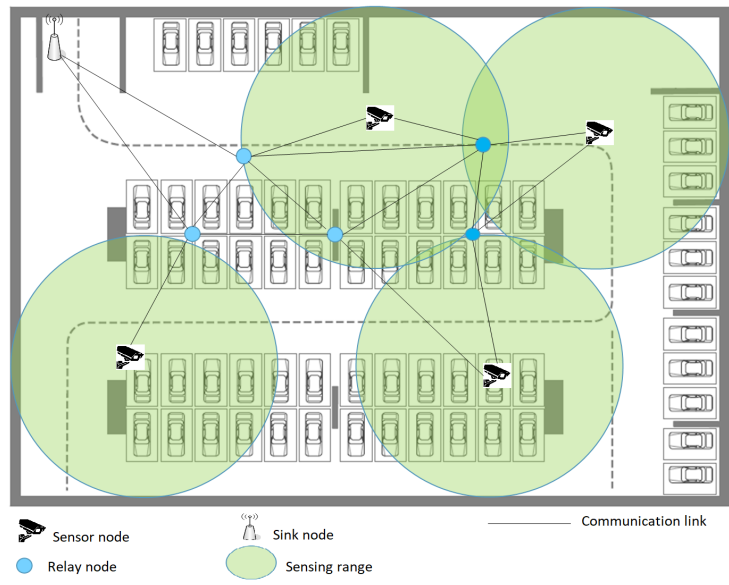

Fig. 1. Example of WSN deployed inside a smart car park

\section{PROBLEM FORMULATION}

Using a discretization of the area of interest (car park space), we obtain a set of points, denoted $\mathcal{P}$. Let $\mathcal{T}=$ $\left\{T_{1}, T_{2}, T_{3}, \ldots, T_{k}\right\}$ be the set of $k$ targets (car park spots) distributed inside the area of interest, which we want to cover by SNs. Each sensor is characterized by a sensing range $R_{s}$, assumed to be constants. SNs and RNs can be placed only at specific candidate sensor positions (CSPs), $\mathrm{CSP}=\left\{s n_{1}, s n_{2}, s n_{3}, \ldots, s n_{n}\right\}$ and candidate relay positions (CRPs), $\mathrm{CRP}=\left\{s r_{1}, s r_{2}, s r_{3}, \ldots ., s r_{m}\right\}$, respectively. Sensor $s n_{j}$ covers the target $T_{i}$ if the sensing range of $s n_{j}$ completely covers the target $T_{i}$. In other words, if the euclidean distance between the sensor $s n_{j}$ and the target $T_{i}$ is no more than $R_{s}$ then the target $T_{i}$ is assumed to be covered by $s n_{j}$. On the other hand, two nodes are neighbors and can communicate (send/receive information) if the Received Signal Strength Indicator (RSSI) value perceived by a node is greater than or equal to $R X_{T s h}{ }^{2}$ which is the sensitivity of the receiving antenna. The radio propagation model used in this paper is Log-normal shadowing model [17].

We consider the problem as a graph consisting of the union of sets of n CSPs, m CRPs and one sink $V_{1}$. Each sensor $i$ has a list $s_{i}$ of targets that can monitor them. The objectives are to minimize:

- The number of sensor nodes, i.e., find $\left|V_{s}^{\text {opt }}\right|$

- The number of relay nodes, i.e., find $\left|V_{c}^{o p t}\right|$

- The Maximum distance of the shortest paths (hop-count) between all SNs to sink node, i.e., $\max _{i \in V_{s}^{\text {opt }}}\left\{\operatorname{dist}_{i, V_{1}}\right\}$ :

\footnotetext{
${ }^{2}$ Represents the minimum RSSI threshold for communication
}

under the two following constraints:

- The union of elements associated with nodes of optimal sensor set covers the set of target $T .\left\{s_{i}\right\}=T, \forall i \in V_{s}^{\text {opt }}$

- Every sensor not neighbor of the sink must be connected to the sink node using at least one path composed only of RNs (Two-tiered architecture [18])

\section{OPTIMIZATION PROBLEM}

In this section, we first give some definitions. Then, we present the MOBILP, and describe the resolution method and how to deal with the objectives.

\section{A. Definitions}

Table II summarizes all of our model's related parameters and variables.

TABLE II

PARAMETERS AND VARIABLES

\begin{tabular}{|c|c|}
\hline \multicolumn{2}{|c|}{ Parameter Definition } \\
\hline Sink & is the index of the sink node \\
\hline$V_{s}$ & is the set of candidate positions of SNs. \\
\hline$V_{r}$ & is the set of candidate positions of RNs. \\
\hline$P$ & is the set of targets. \\
\hline$V_{s}^{p}$ & $\begin{array}{l}\left\{i / \text { Euclidian_distance }(i, p) \leq R_{s} ; i \in V_{s} ; p \in P\right\} \text { is the } \\
\text { set of candidate positions of SNs which cover the target } p \text {. }\end{array}$ \\
\hline$V_{i}^{r}$ & $\begin{array}{l}\left\{j / \quad\left(\left(R S S I(i, j) \geq R X_{T s h}\right) \wedge(R S S I(j, i) \geq\right.\right. \\
\left.\left.\left.R X_{T s h}\right)\right) \wedge\left(j \in\left(V_{r} \vee\{S i n k\}\right)\right)\right\} \text { is the set of candidate } \\
\text { positions of RNs or sink position which are neighbors with } \\
\text { the candidate position of node } i \in\left(V_{s} \cup V_{r}\right) .\end{array}$ \\
\hline$V^{1}$ & $\begin{array}{l}\left\{j /\left(\left(R S S I(\operatorname{Sink}, j) \geq R X_{T s h}\right) \wedge(R S S I(j, \operatorname{Sink}) \geq\right.\right. \\
\left.R X_{T s h}\right) \wedge\left(j \in\left(V_{r} \vee V_{s}\right)\right) \text { is the set of candidate positions } \\
\text { of SNs and RNs which are neighbors with the sink node. }\end{array}$ \\
\hline $\bar{L}$ & Maximum number of hop-count allowed \\
\hline \multicolumn{2}{|c|}{ Variables } \\
\hline$x_{i}{ }^{k}$ & $\begin{array}{l}\text { if a } \mathrm{SN} \text { is placed at candidate position of sensor node } i \text {, and } \\
\text { situated at } \mathrm{k} \text {-hop from the sink node. }\end{array}$ \\
\hline$r_{i}{ }^{k}$ & $\begin{array}{l}\text { if a } \mathrm{RN} \text { is placed at candidate position of relay node } i \text {, and } \\
\text { situated at } \mathrm{k} \text {-hop from the sink node. }\end{array}$ \\
\hline$y_{k}$ & if there is a path with length k-hop from RN to the sink node. \\
\hline
\end{tabular}

\section{B. Multi-objective binary integer linear program}

Using the above parameters and decision variables, the linear model can be written as follows:

$$
\begin{gathered}
\min F 1=\sum_{i \in V_{s}} \sum_{k=1}^{K+1} x_{i}^{k} \\
\min F 2=\sum_{i \in V_{r}} \sum_{k=1}^{K} r_{i}^{k} \\
\min F 3=\sum_{k=1}^{K} y_{k}
\end{gathered}
$$


Subject to:

$$
\begin{aligned}
& \sum_{k=1}^{K+1} x_{i}^{k} \leq 1 \quad i \in V_{s} \\
& \sum_{k=1}^{K} r_{i}^{k} \leq 1 \quad i \in V_{r} \\
& \sum_{k=1}^{K+1} \sum_{i \in V_{s}^{p}} x_{i}^{k} \geq 1 \quad \forall p \in P \\
& x_{i}^{k} \leq \sum_{j \in V_{i}^{r}} r_{j}^{k-1} \quad \forall i \in V_{s} \backslash V^{1}, \forall k=2 \ldots K+1 \\
& r_{i}^{k} \leq \sum_{j \in V_{i}^{r}} r_{j}^{k-1} \quad \forall i \in V_{r} \backslash V^{1}, \quad \forall k=2 \ldots K \\
& \sum_{k=2}^{K+1} x_{i}^{k} \leq 0 \quad i \in V^{1} \backslash V_{r} \\
& \sum_{k=2}^{K} r_{i}^{k} \leq 0 \quad i \in V^{1} \backslash V_{s} \\
& x_{i}^{1}=0 \quad i \in V_{s} \backslash V^{1} \\
& r_{i}^{1}=0 \quad i \in V_{r} \backslash V^{1} \\
& y_{k} \geq r_{i}^{k} \quad i \in V_{r}, \quad k=1 \ldots K \\
& y_{k} \leq \sum_{i \in V_{r}} r_{i}^{k} \quad k=1 \ldots K \\
& y_{k} \leq y_{k-1} \quad k=2 \ldots K \\
& y_{k} \leq \sum_{l=k+1}^{K+1} \sum_{i \in V_{s}} x_{i}^{l} \quad k=1 \ldots K
\end{aligned}
$$

The objective is to minimize simultaneously, F1: number of SNs, F2: number of RNs and F3: diameter of the network.

The constraints (1) and (2) ensure that each SN and RN has only one path to the sink node. The constraint (3) ensures that each target $p \in P$ is covered by at least one $\mathrm{SN}$ which is located at $k$ hops from the sink node. The constraints (4) and (5) guarantees that each $\mathrm{SN}$ or $\mathrm{RN}$ located at $k$ hops from the sink $(k>1)$, has at least one neighbor RN located at $k-1$ hops from the sink node. The constraints (6) and (7) guarantee that a SN or RN respectively, directly neighbor to the sink node cannot be situated at $k>1$ hop. The constraints (8) and (9) avoid the inconsistency of the model, that is to say that no SN and no $\mathrm{RN}$ respectively is neighbor to the sink node unless it is located at 1-hop from it.

The constraint (10) determines the length of the paths made up of RNs towards the sink node. If the $i^{t h}$ relay located at $k$ hops from the sink then there is a path of length $\mathrm{k}$. The constraint (11) ensures that if there is a path of length $k$ hops, it means that there is at least one RN located at $k$ hops from the sink node. This is to avoid the inconsistency of the model. The constraint (12) guarantees that the existence of a path of length $k$ hops induces the presence of a path of length $k-1$ hops. The constraint (13) guarantees that if there is a path with $k$ hops length, a SN is necessarily selected at $l$ hops from sink node $(l=(k+1) \vee l=(k+2) \vee \ldots \vee l=(K+1))$.

\section{Resolution method}

To produce the set of effective solutions, we choose the $\epsilon$-constraint approach[3], which has the advantage of being extremely simple to implement. As shown in algorithm 1, this method consists in transforming the multi-objective problem to a mono-objective problem by considering one objective to optimize among the others and making the remaining objectives as bounded constraints.

We solve the linear program iteratively (line 4), where we minimize the objective function F1 while the objective functions F2 and F3 are considered as constraints delimited respectively by $\epsilon_{2}$ and $\epsilon_{3}$. For each iteration, we increase the bounds $\left(\epsilon_{2}\right.$ or $\left.\epsilon_{3}\right)$ to obtain a new solution.

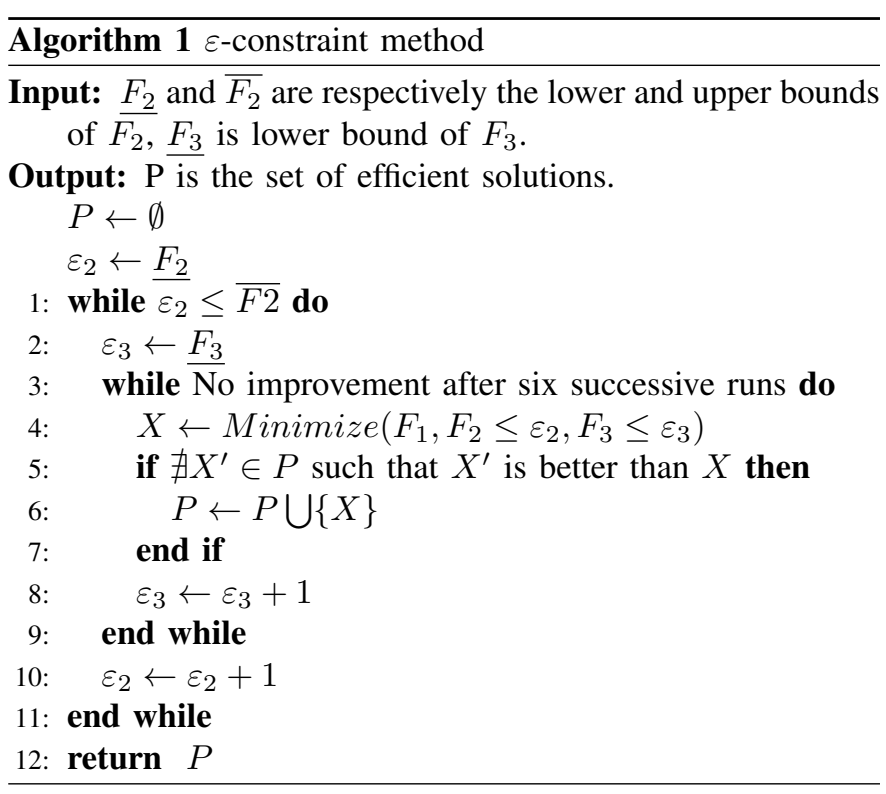

\section{EXPERIMENTAL FINDINGS}

To evaluate the performance of the proposed MOBILP, we conducted extensive tests built on several scenarios. In this section, we describe the considered testbed, the experiments and discuss their results.

\section{A. TESTBED SETUP}

To simulate a test environment corresponding to a car park, we consider a square area of $160 \mathrm{~m} \times 160 \mathrm{~m}$ divided into an equally-sized mesh grid of $20 \mathrm{~m} \times 20 \mathrm{~m}$. We define the CSPs set as the vertices of the whole meshes, the CRPs set as the center of each mesh, and the sink position is picked from the set of CRPs. All nodes have the same Tx power of -5 $\mathrm{dbm}$ and radio sensitivity of $-100 \mathrm{dbm}$, and all SNs have the same sensing range of 25 meters. Three scenarios are studied to show the effectiveness of our model MOBILP denoted Approach 3, compared to research work in [12] and [8] using sequential deployment approach denoted Approach 2, and research work in [15] using mono-objective simultaneously deployment approach denoted Approach 1.

At this stage of research work, and in the absence of real data, we generate the following scenarios: Scenario 1, 
Random distribution of all targets in the area of interest, Scenario 2, Random distribution of $60 \%$ of targets in the left bottom quarter of the grid (near to the car park entrance in bottom left of the grid) and the remaining $40 \%$ is distributed randomly on the other three quarters, and Scenario 3, Random distribution of $60 \%$ of targets in the three first columns of the grid and the remaining $40 \%$ is distributed randomly on the other columns. We vary the sink location for each scenario in the set $\{$ top left, center, bottom right $\}$. For each sink location, the number of targets varies and takes the specified values of 50, 100, and 200 targets. Figure 2 depicts a few generated scenarios built from 200 targets and where the sink is located in the top left of the grid. The mathematical model is solved using " $\epsilon$-Constraint algorithm" in which we call the solver Gurobi 9.0.3 [13], and executed on a PC with a two-core Intel Core Processor $(2.50 \mathrm{GHz})$ and $6 \mathrm{~GB}$ RAM.

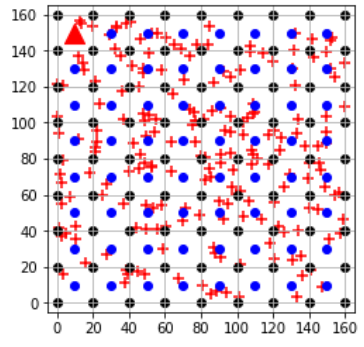

(a) Scenario 1

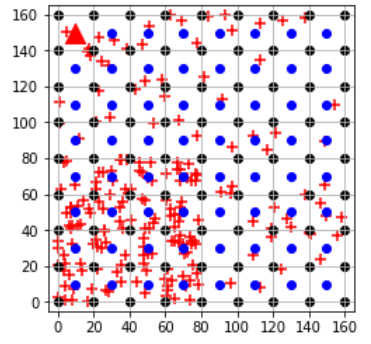

(b) Scenario 2

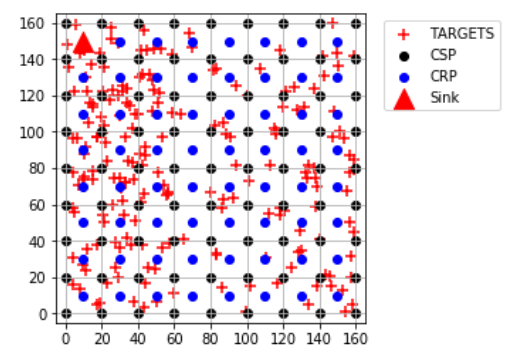

(c) Scenario 3

Fig. 2. The study scenarios: distribution of 200 targets with the sink in top left

\section{B. RESULTS AND DISCUSSION}

We carried out several tests, the outcomes from program runs are depicted in tables III, IV and V. In comparison to Approach 1, that is the mono-objective optimization with simultaneous placement of SNs and RNs, MOBILP provides a set of alternative solutions which include the solution obtained from the Approach 1. On the other hand, we observe that all the solutions provided by Approach 2 are also included in the set of solutions provided by MOBILP or are dominated by those solutions. Therefore, modelling a simultaneous deployment problem as a multi-objective problem allows the decision-maker more flexibility to choose the optimal trade-off between conflicting objectives according to the context of the problem. Thus, the decision-maker can pick any specific number of relay nodes (\#RN) and obtain the minimum associated number of SNs (\#SN) and network diameter (D).
TABLE III

TEST RESULTS: SINK AT THE TOP LEFT

\begin{tabular}{|c|c|c|c|c|c|c|c|c|c|}
\hline & \multicolumn{3}{|c|}{ Scenario 1} & \multicolumn{3}{|c|}{ Scenario 2} & \multicolumn{3}{|c|}{ Scenario 3} \\
\hline & \#SN & \#RN & $\mathrm{D}$ & \#SN & \#RN & $\mathrm{D}$ & \#SN & \#RN & $\mathrm{D}$ \\
\hline & \multicolumn{9}{|c|}{50 target } \\
\hline App1 & 18 & 12 & 7 & 13 & 10 & 6 & 19 & 11 & 7 \\
\hline App2 & 18 & 13 & 7 & 13 & 11 & 6 & 19 & 12 & 7 \\
\hline \multirow{3}{*}{ MOBILP } & 18 & 12 & 7 & 13 & 9 & 8 & \multirow{2}{*}{19} & \multirow{2}{*}{11} & \multirow{2}{*}{7} \\
\hline & \multirow{2}{*}{19} & \multirow{2}{*}{11} & \multirow{2}{*}{7} & \multirow{2}{*}{$\begin{array}{l}13 \\
14\end{array}$} & \multirow{2}{*}{$\begin{array}{l}10 \\
99\end{array}$} & \multirow{2}{*}{$\begin{array}{l}6 \\
7\end{array}$} & & & \\
\hline & & & & & & & 20 & 13 & 6 \\
\hline & \multicolumn{9}{|c|}{100 targets } \\
\hline App1 & 23 & 13 & 7 & 21 & 11 & 7 & 22 & 12 & 7 \\
\hline App2 & 23 & 13 & 7 & 21 & 12 & 7 & 21 & 13 & 7 \\
\hline \multirow{4}{*}{ MOBILP } & 23 & 12 & 8 & \multirow{4}{*}{21} & \multirow{4}{*}{11} & \multirow{4}{*}{7} & \multirow{2}{*}{21} & \multirow{2}{*}{12} & \multirow{2}{*}{7} \\
\hline & 23 & 13 & 7 & & & & & & \\
\hline & 24 & 12 & 7 & & & & 22 & 11 & 7 \\
\hline & 25 & 11 & 8 & & & & & & 1 \\
\hline & \multicolumn{9}{|c|}{200 targets } \\
\hline App1 & 33 & 11 & 7 & 26 & 13 & 6 & 30 & 14 & 7 \\
\hline \multirow{2}{*}{ App2 } & 31 & 15 & 8 & 26 & 12 & 9 & 30 & 15 & 8 \\
\hline & 31 & 16 & 7 & 26 & 13 & 6 & 30 & 16 & 7 \\
\hline \multirow{6}{*}{ MOBILP } & 31 & 13 & 8 & 26 & 11 & 8 & 30 & 13 & 8 \\
\hline & 31 & 14 & 7 & 26 & 12 & 7 & 30 & 14 & 7 \\
\hline & 32 & 12 & 7 & 26 & 13 & 6 & 31 & 13 & 7 \\
\hline & \multirow{2}{*}{32} & \multirow{2}{*}{13} & \multirow{2}{*}{6} & 27 & 11 & 7 & 31 & 14 & 6 \\
\hline & & & & 27 & 12 & 6 & 32 & 12 & 7 \\
\hline & 33 & 11 & 7 & 28 & 10 & 8 & 32 & 13 & 6 \\
\hline
\end{tabular}

TABLE IV

TEST RESUlTS: SinK AT THE CENTRE

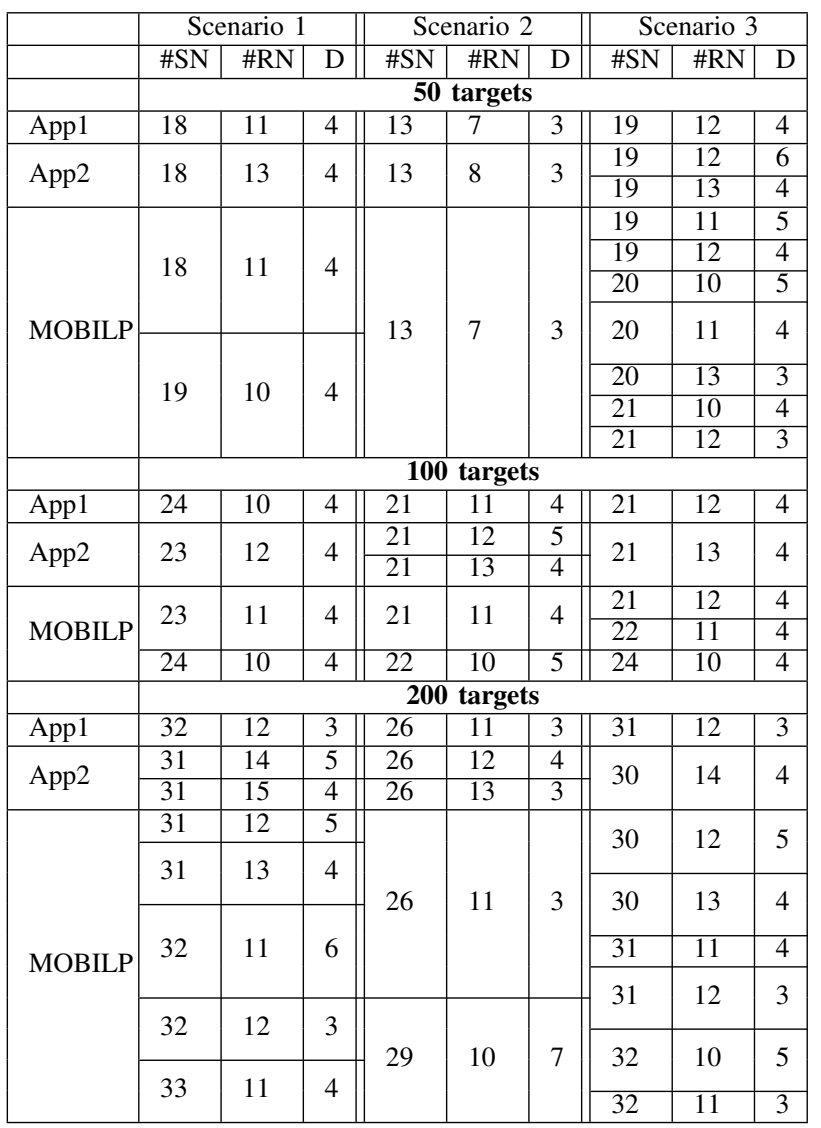


TABLE V

TEST RESUlTS: SinK AT THE BOTTOM RIGHT

\begin{tabular}{|c|c|c|c|c|c|c|c|c|c|}
\hline & \multicolumn{3}{|c|}{ Scenario 1} & \multicolumn{3}{|c|}{ Scenario 2} & \multicolumn{3}{|c|}{ Scenario 3} \\
\hline & \#SN & \#RN & $\mathrm{D}$ & \#SN & \#RN & $\mathrm{D}$ & \#SN & \#RN & $\mathrm{D}$ \\
\hline & \multicolumn{9}{|c|}{50 targets } \\
\hline App1 & 18 & 11 & 5 & 13 & 8 & 4 & 19 & 12 & 5 \\
\hline \multirow{3}{*}{ App2 } & 18 & 13 & 7 & \multirow{2}{*}{13} & \multirow{2}{*}{8} & \multirow{2}{*}{6} & \multirow{2}{*}{19} & \multirow{2}{*}{12} & \multirow{2}{*}{6} \\
\hline & 18 & 14 & 6 & & & & & & \\
\hline & 18 & 15 & 5 & 13 & 9 & 4 & 19 & 13 & 5 \\
\hline \multirow{3}{*}{ MOBILP } & 18 & 11 & 5 & 13 & 8 & 4 & 19 & 12 & 5 \\
\hline & & & $J$ & 15 & 0 & 4 & 20 & 11 & 5 \\
\hline & 19 & 10 & 7 & 15 & 7 & 5 & 21 & 10 & 8 \\
\hline & \multicolumn{9}{|c|}{100 target } \\
\hline App1 & 23 & 12 & 5 & 22 & 11 & 5 & 21 & 13 & 5 \\
\hline \multirow{3}{*}{ App2 } & 23 & 11 & 9 & \multirow{2}{*}{21} & \multirow{2}{*}{11} & \multirow{2}{*}{6} & \multirow{2}{*}{21} & \multirow{2}{*}{12} & \multirow{2}{*}{7} \\
\hline & 23 & 12 & 6 & & & & & & \\
\hline & 23 & 14 & 5 & 21 & 12 & 5 & 21 & 13 & 5 \\
\hline \multirow{6}{*}{ MOBILP } & \multirow{2}{*}{23} & \multirow{2}{*}{11} & \multirow{2}{*}{7} & \multirow{2}{*}{21} & \multirow{2}{*}{11} & \multirow{2}{*}{6} & 21 & 12 & 6 \\
\hline & & & & & & & 21 & 13 & 5 \\
\hline & 23 & 12 & 5 & 21 & 12 & 5 & 22 & 11 & 6 \\
\hline & \multirow[t]{2}{*}{24} & 11 & 5 & 22 & 10 & 7 & 23 & 12 & 5 \\
\hline & & & & & & & & 10 & 6 \\
\hline & 25 & 10 & 0 & 22 & 11 & 5 & 25 & 11 & 5 \\
\hline & & & & & $\operatorname{targe}$ & & & & \\
\hline App1 & 31 & 13 & 5 & 26 & 12 & 5 & 30 & 12 & 5 \\
\hline & 31 & 13 & 8 & & & & 30 & 14 & 7 \\
\hline App2 & & & & 26 & 13 & 5 & 30 & 15 & 6 \\
\hline & 31 & 14 & 5 & & & & 30 & 16 & 5 \\
\hline & 31 & 12 & 8 & & & & 30 & 12 & 5 \\
\hline & 31 & 13 & 5 & 20 & 12 & 5 & & & \\
\hline MOBIL P & 31 & 11 & 8 & & & & 31 & 11 & 6 \\
\hline & & & & & & & & & \\
\hline & & & & & & & 32 & 11 & 5 \\
\hline & 33 & 11 & 6 & 28 & 10 & 5 & & & \\
\hline
\end{tabular}

There is an impact of target density as shown in Tables III, IV and V. We note that when the density of targets increases inside the smart parking, more SNs and RNs are required, and hence the cost of the deployment increases significantly. This can be explained by the limited sensing range of the SNs which reduces the number of SNs that cover the same target and leads to the increase of the number of SNs and RNs to ensure coverage of all targets and connectivity requirements. Furthermore, the variation of the distribution of the target positions according to the three scenarios detailed above has an impact on the WSN deployment as shown in Tables III, IV and V. Indeed, in scenario 2 when all the targets are close as they are concentrated in one area, the \#SN has the lowest value. This is because one SN can cover more targets.

In addition, as illustrated in Tables III, IV and V, it appears that the sink position affects only the diameter of the network whatever the scenario. We can notice that the best position of the sink to get the better value of the diameter is in the middle of the area under study, as shown in the Table IV, where all SNs are near the sink node. Finally, we can conclude that MOBILP generally outperforms the other approaches or at least provides the same solutions in all scenarios.

On the other hand, MOBILP takes longer running time compared to the other two approaches. For instance, In scenario 1 , when the number of targets is 50 the approaches 1,2 and MOBILP solve the problem in $2.67 \mathrm{~s}, 3.02 \mathrm{~s}$ and $35.88 \mathrm{~s}$ respectively. While in scenario 2 they solve the problem in $5.54 \mathrm{~s}, 3.95 \mathrm{~s}$ and $21.27 \mathrm{~s}$. Finally, for scenario 3 they take $2.20 \mathrm{~s}$, $2.29 \mathrm{~s}$ and $15.32 \mathrm{~s}$ respectively. This can be explained by the simultaneous deployment that increases the research space of the problem and the multi-objective version that makes the problem more complex. On the other side, the running time of the MOBILP increases when the number of targets increases. For example, to solve scenario 1 with 50, 100 and 200 targets the MOBILP takes 35.88s, 117.61s and 598.92s respectively. Nevertheless, we can agree on a reasonable resolution time as we are working offline. Indeed, we have considered the car park spots as the targets, not the vehicles.

\section{CONCLUSIONS AND FUTURE WORK}

In this work, we addressed the optimal deployment of WSNs for fire detection in a smart car park by identifying the locations of SNs and RNs simultaneously, minimizing the number of SNs, the number of RNs and the network diameter while satisfying the coverage and connectivity constraints. First, we formulated the problem as a graph, then we solved it using our approach referred to as "MOBILP".

We also performed exhaustive testing on three scenarios. The first one is purely random. The two other scenarios are close to reality, where the first one has $60 \%$ of the targets distributed in the lower left quarter near the smart parking entrance and $40 \%$ distributed randomly in the three other quarters, and the second one has $60 \%$ of the targets in the three first columns near the smart parking entrance and $40 \%$ distributed randomly on the other remaining columns. The MOBILP provides better solutions and more flexibility to choose the optimal compromise between contradictory objectives, in comparison to two existing approaches, a sequential approach which places SNs then RNs, and an approach of simultaneous deployment of SNs and RNs with mono-objective function that provides only one solution.

In our future work, we intend to address a wider car park area and we will move towards the use of heuristics to solve large instances of our problem within a reasonable time.

\section{REFERENCES}

[1] S. Abdollahzadeh and Nima Jafari Navimipour. "Deployment strategies in the wireless sensor network: A comprehensive review". In: Comput. Commun. 91-92 (2016), pp. 1-16.

[2] A. H. Alavi et al. "Internet of Things-enabled smart cities: State-of-the-art and future trends". In: Measurement 129 (2018).

[3] Ricardo Landa Becerra and Carlos A. Coello Coello. "Solving Hard Multiobjective Optimization Problems Using $\epsilon$-Constraint with Cultured Differential Evolution". In: ed. by Thomas Philip Runarsson et al. Springer Berlin Heidelberg, 2006.

[4] J. L. Bredin et al. "Deploying Sensor Networks With Guaranteed Fault Tolerance". In: IEEE/ACM Transactions on Networking 18.1 (2010), pp. 216-228. 
[5] Soumaya Fellah and Mejdi Kaddour. "Exact and Efficient Heuristic Deployment in WSN under Coverage, Connectivity, and Lifetime Constraints". In: International Journal of Mobile Computing and Multimedia Communications (IJMCMC) 8.2 (2017).

[6] Xiuming Guo et al. "A Deterministic Sensor Node Deployment Method with Target Coverage and Node Connectivity". In: Artificial Intelligence and Computational Intelligence. Ed. by Hepu Deng et al. Springer Berlin Heidelberg, 2011, pp. 201-207.

[7] Govind P Gupta and Sonu Jha. "Biogeography-based optimization scheme for solving the coverage and connected node placement problem for wireless sensor networks". In: Wireless Networks 25.6 (2019).

[8] Nguyen Thi Hanh et al. "Minimal Node Placement for Ensuring Target Coverage With Network Connectivity and Fault Tolerance Constraints in Wireless Sensor Networks". In: 2019 IEEE Congress on Evolutionary Computation (CEC).

[9] M. Y. I. Idris et al. "Smart Parking System using Image Processing Techniques in Wireless Sensor Network Environment". In: Information Technology Journal 8 (2009), pp. 114-127.

[10] Arpit Jadon, Akshay Varshney, and Mohammad Samar Ansari. "Low-Complexity High-Performance Deep Learning Model for Real-Time Low-Cost Embedded Fire Detection Systems". In: Procedia Computer Science 171 (2020). Third International Conference on Computing and Network Communications (CoCoNet'19), pp. 418-426.

[11] T. Lin, H. Rivano, and F. Le Mouël. "A Survey of Smart Parking Solutions". In: IEEE Transactions on Intelligent Transportation Systems 18.12 (2017), pp. 3229-3253.

[12] Arouna Ndam Njoya et al. "Hybrid Wireless Sensors Deployment Scheme with Connectivity and Coverage Maintaining in Wireless Sensor Networks". In: Wireless Personal Communications (2020).

[13] Gurobi Optimization. Inc., "Gurobi optimizer reference manual," 2015.

[14] "Performance Analysis of Proximity and Light Sensors for Smart Parking". In: Procedia Computer Science 83 (2016), pp. 385-392.

[15] Y. Sun and S. Halgamuge. "Minimum-Cost Heterogeneous Node Placement in Wireless Sensor Networks". In: IEEE Access 7 (2019), pp. 14847-14858.

[16] Y. Sun et al. "A Physarum-Inspired Algorithm for Minimum-Cost Relay Node Placement in Wireless Sensor Networks". In: IEEE/ACM Transactions on Networking 28.2 (2020), pp. 681-694.

[17] Lixin Wang, Peng-Jun Wan, and William Washington. "Connectivity of multihop wireless networks with lognormal shadowing”. In: Wireless Networks 21.7 (2015), pp. 2279-2292.

[18] Dejun Yang et al. "Two-Tiered Constrained Relay Node Placement in Wireless Sensor Networks: Computational Complexity and Efficient Approximations". In:
IEEE Transactions on Mobile Computing 11.8 (2012), pp. 1399-1411. 